\title{
Application of patch stimulator for intraoperative neuromonitoring during thyroid surgery: maximizing surgeon's convenience
}

\author{
Moon Young $\mathrm{Oh}^{1} \wedge$, Jung-Man Lee ${ }^{2,3}$, Myung-ho Lee ${ }^{4}$, Hyun Suk Choi ${ }^{5}$, Jongjin Kim ${ }^{1,4}$, Ki-Tae Hwang ${ }^{1,4}$, \\ Young Jun Chai ${ }^{1,4,6}$ \\ ${ }^{1}$ Department of Surgery, Seoul National University College of Medicine, Seoul, Korea; ${ }^{2}$ Department of Anesthesiology and Pain Medicine, Seoul \\ National University College of Medicine, Seoul, Korea; ${ }^{3}$ Department of Anesthesiology and Pain Medicine, Seoul Metropolitan Government - Seoul \\ National University Boramae Medical Center, Seoul, Korea; ${ }^{4}$ Department of Surgery, Seoul Metropolitan Government - Seoul National University \\ Boramae Medical Center, Seoul, Korea; ${ }^{5}$ Department of Nursing, Seoul Metropolitan Government - Seoul National University Boramae Medical \\ Center, Seoul, Korea; ${ }^{6}$ Transdisciplinary Department of Medicine \& Advanced Technology, Seoul National University Hospital, Seoul, Korea \\ Contributions: (I) Conception and design: MY Oh, YJ Chai; (II) Administrative support: YJ Chai, J Kim, KT Hwang; (III) Provision of study \\ materials or patients: YJ Chai, JM Lee; (IV) Collection and assembly of data: All authors; (V) Data analysis and interpretation: MY Oh, YJ Chai; (VI) \\ Manuscript writing: All authors; (VII) Final approval of manuscript: All authors. \\ Correspondence to: Young Jun Chai, MD, PhD. Department of Surgery, Seoul National University College of Medicine, Seoul Metropolitan \\ Government - Seoul National University Boramae Medical Center, Transdisciplinary Department of Medicine \& Advanced Technology, Seoul \\ National University Hospital, 20 Boramae-ro 5-gil, Dongjak-gu, Seoul 07061, Korea. Email: kevinjoon@naver.com.
}

\begin{abstract}
Background: Intraoperative neuromonitoring (IONM) is frequently used in thyroid surgery to reduce recurrent laryngeal nerve (RLN) injury by providing the surgeon with real-time feedback on nerve stimulation during dissection. We applied a disposable adhesive patch electrode to a dissecting instrument to transfer electrical stimulation to the dissecting instrument for IONM during thyroid surgery. This study aimed to evaluate the feasibility of using the patch stimulator approach for IONM during thyroid surgery.

Methods: We reviewed the medical records of patients who underwent thyroidectomy using both conventional stimulator and adhesive patch stimulator for IONM. The electromyography (EMG) amplitudes of the vagal and the RLNs before (V1, R1) and after thyroid resection (V2, R2) were alternatively checked with each type of stimulator at the same location of each nerve.

Results: Fifteen consecutive patients (4 males, 11 females) were included in this analysis, and a total of 38 nerves (19 vagus nerves and 19 RLNs) were evaluated. No statistically significant differences were seen in the mean amplitudes evoked by the patch stimulator and the conventional probe stimulator for the V1 signal (825.5 \pm 394.6 vs. $821.8 \pm 360.9 \mu \mathrm{V}, \mathrm{P}=0.954)$, $\mathrm{R} 1$ signal $(1,044.8 \pm 471.2$ vs. $1,039.2 \pm 507.4 \mu \mathrm{V}, \mathrm{P}=0.898), \mathrm{R} 2$ signal $(1,037.8 \pm 495.0$ vs. $938.2 \pm 415.8 \mu \mathrm{V}, \mathrm{P}=0.948)$, or $\mathrm{V} 2$ signal $(812.5 \pm 391.9$ vs. $787.3 \pm 355.7 \mu \mathrm{V}, \mathrm{P}=0.975)$.

Conclusions: The patch stimulator was safely and effectively used for IONM during thyroid surgery and provided similar nerve monitoring responses as the conventional stimulator. This approach may be used to enhance the surgeon's convenience during thyroid surgery.
\end{abstract}

Keywords: Neuromuscular monitoring; electrodes; electromyography (EMG); thyroidectomy

Submitted Jun 01, 2021. Accepted for publication Jul 22, 2021.

doi: $10.21037 / g s-21-327$

View this article at: https://dx.doi.org/10.21037/gs-21-327

$\wedge$ ORCID: 0000-0002-8939-1735. 


\section{Introduction}

Recurrent laryngeal nerve (RLN) injury is a highly undesirable complication during thyroid surgery because it can lead to hoarseness, dysphonia, dysphagia, pulmonary aspiration, and life-threatening airway obstruction $(1,2)$. Intraoperative neuromonitoring (IONM) is increasingly being used routinely in thyroid surgery, as it has been shown to prevent RLN injury by facilitating identification of the nerve anatomically, as well as enabling recognition of anatomic variants, leading to preservation of the nerve's functional integrity $(3,4)$.

Traditional intermittent IONM (I-IONM) is performed using a conventional nerve stimulator, which is a handheld probe that stimulates the potential target structure intermittently during surgery (5). Using this technique, the function of the RLN can only be evaluated at the moment of stimulation, and thus, the nerve is still at risk of injury during the time gap between stimulations. Furthermore, the need to repeatedly shift between surgical instruments and the conventional stimulator is troublesome for the surgeon and time-consuming.

Recently, new monitoring tools and methods have been developed to solve the problems of I-IONM and to enhance both the surgeon's convenience and patient safety. Stimulating dissecting instruments with combined functionality were developed with the ability to perform both dissection and nerve stimulation. The first proposed method was to tape stimulation electrical wires directly to the dissecting tools $(6,7)$. Many other variations of stimulating dissecting instruments have been proposed thereafter, such as detachable magnetic stimulators and attachable ring stimulators $(8,9)$. However, we noticed that these approaches each have their own limitations, including being too costly, too bulky, or prone to detachment from dissecting tools.

To overcome the disadvantages of these instruments, we devised an adhesive patch stimulator approach to IONM. An adhesive patch electrode commonly used in transcutaneous electrical nerve stimulation therapies (Surface Electrode Ambu Neuroline 700 15-K/C/12, Ambu, Copenhagen) was attached to a dissecting instrument to transfer electrical stimulation to the dissecting instrument. The adhesive patch electrode is small $\left(20 \times 15 \times 1 \mathrm{~mm}^{3}\right)$, lightweight $(4$ grams), inexpensive (US \$2.5), disposable, and easily applied onto dissecting instruments. The aim of this study is to evaluate the feasibility of using the patch stimulator for IONM during thyroid surgery, by comparing the electromyography (EMG) amplitudes of nerves stimulated by a patch stimulator and a conventional stimulator.

We present the following article in accordance with the STROBE reporting checklist (available at https://dx.doi. org/10.21037/gs-21-327).

\section{Methods}

\section{Patients}

We used both a conventional stimulator and the patch stimulator for nerve stimulation for IONM during each thyroid surgery at Seoul Metropolitan Government Seoul National University Boramae Medical Center from April 5, 2021 to April 30, 2021. We retrospectively reviewed the IONM records of all patients who underwent thyroid surgery during the study period. The study was conducted in accordance with the Declaration of Helsinki (as revised in 2013). This study was approved by the Institutional Review Board of the Seoul Metropolitan Government Seoul National University Boramae Medical Center (IRB number: 30-2021-53) and individual consent for this retrospective analysis was waived.

\section{Anesthesia and monitoring setup}

Anesthesia was induced with lidocaine $(30 \mathrm{mg})$ and propofol $(1.5 \mathrm{mg} / \mathrm{kg})$ injections, then maintained with targetcontrolled infusions of propofol and remifentanil using infusion pumps. Rocuronium $(0.6 \mathrm{mg} / \mathrm{kg})$ was administered for muscle relaxation. A surgical thyroid pillow was used for positioning the patient prior to intubation to prevent inadvertent tube displacement. Endotracheal intubation was performed using an EMG endotracheal tube (Medtronic, Jacksonville, FL, USA). The surface electrodes on the tube were placed at the level of the vocal cords. Immediately after tube fixation, neostigmine $(2 \mathrm{mg})$ and glycopyrrolate $(0.4 \mathrm{mg})$ were administered for the reversal of neuromuscular blockade. One anesthesiologist (J.L) performed or supervised all anesthetic procedures.

Equipment setup and nerve monitoring were performed following the standardized procedures of the International Neural Monitoring Study Group (INMSG) guidelines (10). Nerve integrity monitoring (NIM) was performed using the NIM-response 3.0 system (Medtronic, Jacksonville, Florida). The stimulus current was set to $1 \mathrm{~mA}$ with a frequency of $4 \mathrm{~Hz}$, the event threshold was set to $100 \mathrm{mV}$, and the stimulation duration was set to $100 \mathrm{~ms}$. The cutoff 


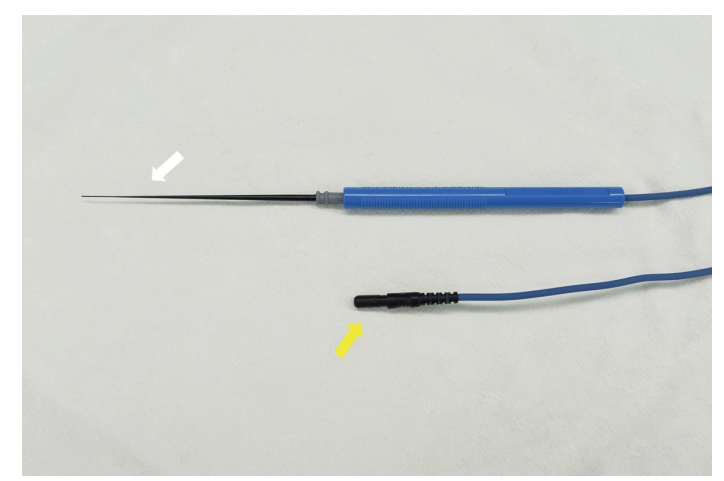

Figure 1 Conventional stimulator. Handheld probe tip (white arrow) connected to the monitoring system via connector (yellow arrow).

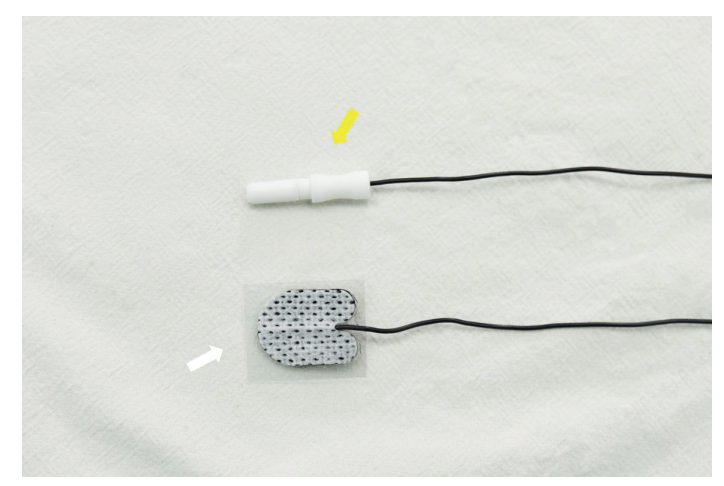

Figure 2 Adhesive patch electrode. Adhesive electrode patch (white arrow) connected to the monitoring system via connector (yellow arrow).

value for the vagus nerve $(\mathrm{VN})$ response and RLN response was set at $100 \mu \mathrm{V}$.

\section{IONM procedures using the conventional stimulator and the patch stimulator}

Both the conventional stimulator and the patch stimulator were used for each operation. The conventional stimulator (Prass Standard Monopolar Stimulator Probe, Medtronic, FL, Figure 1) was connected to the monitoring system and use $\mathrm{d}$ during surgery by stimulating the nerves with the tip of the probe. The patch stimulator (Figure 2) was prepared by wrapping the adhesive electrode pad around one ring of the mosquito forceps used for dissection. After connecting the wires to the monitoring system, nerve monitoring was implemented with the mosquito forceps during dissection (Figure 3).

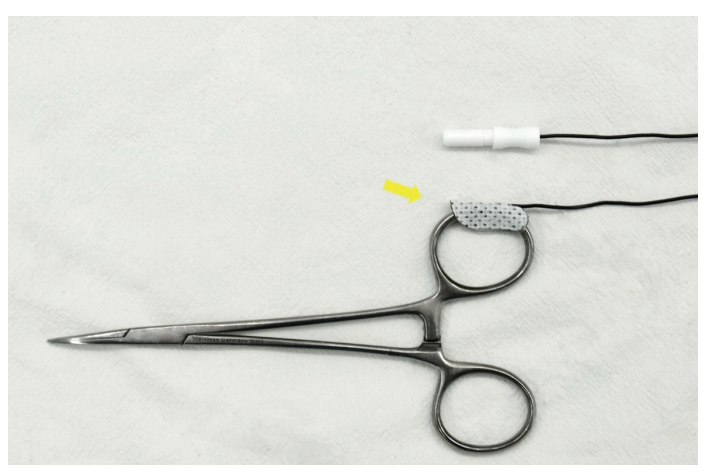

Figure 3 Patch stimulator. An adhesive patch electrode is applied on mosquito forceps (yellow arrow) to transfer electrical stimulus to the nerve through the instrument.

Surgery was performed by a single surgeon (Y.J.C.). $\mathrm{VN}$ and RLN nerve stimulations were performed before and after resection, using both the patch stimulator and the conventional stimulator. The V1 and R1 signals were defined as the EMG signals from the VN and RLN, respectively, upon their initial identification prior to thyroid resection. The VN and RLN were first stimulated by the patch stimulator and then stimulated by the conventional stimulator at the same location of each nerve. The R2 and V2 signals were defined as the EMG signals of the RLN and VN, respectively, after thyroid gland removal. The $\mathrm{VN}$ and RLN were stimulated at the same location of the nerve by the patch stimulator followed by the conventional stimulator alternatively.

\section{Statistical analysis}

All data are presented as mean \pm standard deviation for continuous variables. Statistical analysis was performed by the chi-square test, and two tailed values of $\mathrm{P}<0.05$ were considered statistically significant. All analyses were performed using SPSS 26.0 software for Windows (IBM, Armonk, New York, USA).

\section{Results}

A total of 15 consecutive patients (4 males and 11 females) were included in the study. Patient characteristics are summarized in Table 1 . The mean age was $50.9 \pm 12.0$ years, and the mean body mass index was $25.3 \pm 3.8$. The mean tumor size at the longest diameter was $1.4 \pm 0.9 \mathrm{~cm}$. Eleven (73.3\%) patients underwent lobectomy and four (26.7\%) 
Table 1 Patient characteristics

\begin{tabular}{lc}
\hline Patient characteristics & Total patients $(\mathrm{N}=15)$ \\
\hline Gender (male:female) & $4: 11$ \\
Age (years) & $50.9 \pm 12.0$ \\
Body mass index $\left(\mathrm{kg} / \mathrm{m}^{2}\right)$ & $25.3 \pm 3.8$ \\
Tumor size in longest diameter $(\mathrm{cm})$ & $1.4 \pm 0.9$ \\
Extent of operation & \\
Lobectomy & $11(73.3)$ \\
Total thyroidectomy & $4(26.7)$ \\
Diagnosis & $10(66.7)$ \\
Papillary thyroid carcinoma & $3(20.0)$ \\
Follicular cell adenoma & $1(6.7)$ \\
Hurthle cell adenoma & $1(6.7)$ \\
Graves' disease & $0(0.0)$ \\
Vocal cord palsy &
\end{tabular}

All data are presented as mean \pm standard deviation or as $n(\%)$, unless stated otherwise.

underwent total thyroidectomy. The final pathological diagnoses consisted of 10 cases of papillary thyroid carcinomas, three cases of follicular cell adenomas, one case of Hurthle cell adenomas, and one case of Graves' disease. No vocal cord palsy occurred in any of the patients (Table 1).

Table 2 shows the EMG amplitude profiles of RLN and VN stimulated by the patch stimulator and a conventional probe stimulator. A total of 38 nerves (19 RLNs and 19 $\mathrm{VNs}$ ) were evaluated. When stimulated with each of the stimulators, the V1, R1, R2, and V2 signals of all the nerves had amplitudes of more than $500 \mu \mathrm{V}$, except in one patient who had V1 and R1 signals of more than $500 \mu \mathrm{V}$ but R2 and V2 signals of less than $500 \mu \mathrm{V}$. No postoperative vocal cord palsy was seen in this patient. The mean amplitudes evoked by the patch stimulator and the conventional probe stimulator were $935.8 \pm 426.1$ and $928.0 \pm 414.4 \mu \mathrm{V}$ for the V1 signal, $1,165.6 \pm 537.3$ and $1,143.0 \pm 547.7 \mu \mathrm{V}$ for the $\mathrm{R} 1$ signal, $1,082.8 \pm 522.4$ and $1,071.6 \pm 527.0 \mu \mathrm{V}$ for the $\mathrm{R} 2$ signal, and $872.7 \pm 453.2$ and $877.6 \pm 507.1 \mu \mathrm{V}$ for the V2 signal, respectively. The mean differences between the amplitudes evoked by the two types of stimulators were $70.8 \pm 65.3 \mu \mathrm{V}$ for the $\mathrm{V} 1,70.9 \pm 72.4 \mu \mathrm{V}$ for the $\mathrm{R} 1$, $155.1 \pm 187.1 \mu \mathrm{V}$ for the $\mathrm{R} 2$, and $83.8 \pm 110.2 \mu \mathrm{V}$ for the $\mathrm{V} 2$ signals. There were no statistical differences in the mean amplitudes between the two groups for the V1 $(\mathrm{P}=0.954)$,
R1 ( $\mathrm{P}=0.898), \mathrm{R} 2(\mathrm{P}=0.948)$, and V2 ( $\mathrm{P}=0.975)$ signals.

\section{Discussion}

Although IONM is increasingly being routinely used in thyroid surgery and has become the standard practice in high-risk cases, there is still debate over whether I-IONM reduces RLN injury during thyroid surgery $(11,12)$. RLN injury, recognized by loss of signal, has been reported even with I-IONM. The suggested reason for the loss of signal is that I-IONM does not provide real-time monitoring, thus I-IONM detects nerve injury only after the damage has occurred $(4,13)$. The RLN is still at risk of injury proximally to the site of intermitted stimulation and during the interval between nerve stimulations (14).

Continuous IONM (C-IONM) was subsequently established to overcome the limitations of I-IONM (15). By placing a monopolar automatic periodic stimulation electrode on the VN, constant nerve function monitoring is possible throughout the whole course of surgery, allowing the surgeon to dissect and stimulate at the same time (16). However, C-IONM requires the risky procedure of dissecting the carotid sheath and fully exposing the $\mathrm{VN}$ to place the electrode on the nerve $(17,18)$. Hemodynamic instability and reversible vagal neuropraxia have also been associated with C-IONM, suggesting that this approach may cause patient harm (19). Furthermore, there is still controversy over whether C-IONM actually reduces the risk of RLN injury (20).

Recently, the use of stimulating dissecting instruments that combine the beneficial features of both I-IONM and C-IONM has been proposed. Stimulating dissecting instruments, which are conventional surgical instruments that are connected to a monitoring system by stimulation wires or attachable stimulators, provide the surgeon with an effective way to perform dissection while simultaneously carrying out nerve stimulation (6,7). In addition to realtime nerve monitoring, stimulating dissecting instruments reduce the time interval between stimulations because they do not require the surgeon to constantly switch between the stimulator and the dissecting instrument, unlike conventional I-IONM monopolar probe stimulators.

The first documented stimulating dissecting instruments were prepared by connecting the surgical instruments to the monitoring system with stimulation wires bound to the instruments with a transparent film (6). Afterwards, the application of different types of stimulators, including a magnetic attachable stimulator and an attachable ring 
Table 2 EMG amplitude profiles of recurrent laryngeal nerve and vagus nerve stimulated by a patch stimulator and a conventional stimulator

\begin{tabular}{|c|c|c|c|c|c|c|c|c|c|c|c|c|c|}
\hline $\begin{array}{l}\text { Patient } \\
\text { number }\end{array}$ & Side & \multicolumn{3}{|c|}{$\mathrm{V} 1(\mu \mathrm{V})$} & \multicolumn{3}{|c|}{$\mathrm{R} 1(\mu \mathrm{V})$} & \multicolumn{3}{|c|}{$\mathrm{R} 2(\mu \mathrm{V})$} & \multicolumn{3}{|c|}{$\mathrm{V} 2(\mu \mathrm{V})$} \\
\hline 1 & Rt & 550 & 571 & 21 & 538 & 522 & 16 & 967 & 737 & 230 & 587 & 576 & 11 \\
\hline 1 & $\mathrm{Lt}$ & 627 & 551 & 76 & 929 & 797 & 132 & 1,061 & 1,084 & 23 & 805 & 738 & 67 \\
\hline 2 & Rt & 1,769 & 1,659 & 110 & 1,407 & 1,405 & 2 & 661 & 507 & 154 & 501 & 432 & 69 \\
\hline 3 & $\mathrm{Lt}$ & 523 & 677 & 154 & 1,024 & 1,013 & 11 & 1,194 & 959 & 235 & 718 & 529 & 189 \\
\hline 4 & Rt & 666 & 658 & 8 & 777 & 841 & 64 & 660 & 667 & 7 & 807 & 918 & 111 \\
\hline 5 & $\mathrm{Lt}$ & 567 & 567 & 0 & 1,196 & 1,002 & 194 & 647 & 818 & 171 & 699 & 697 & 2 \\
\hline 6 & Rt & 752 & 690 & 62 & 560 & 575 & 15 & 650 & 633 & 17 & 524 & 715 & 191 \\
\hline 8 & Lt & 561 & 530 & 31 & 675 & 611 & 64 & 915 & 759 & 156 & 542 & 547 & 5 \\
\hline 9 & Rt & 1,386 & 1,300 & 86 & 2,236 & 2,311 & 75 & 2,391 & 2,092 & 299 & 1,942 & 1,801 & 141 \\
\hline 10 & Rt & 1,648 & 1,851 & 203 & 1,977 & 1,998 & 21 & 1,759 & 1,761 & 2 & 1,446 & 1,453 & 7 \\
\hline 10 & $\mathrm{Lt}$ & 689 & 624 & 65 & 1,414 & 1,458 & 44 & 1,125 & 1,071 & 54 & 701 & 615 & 86 \\
\hline 11 & Rt & 819 & 796 & 23 & 844 & 713 & 131 & 862 & 821 & 41 & 888 & 862 & 26 \\
\hline 12 & Lt & 1,513 & 1,461 & 52 & 2,144 & 2,084 & 60 & 2,025 & 1,937 & 88 & 1,025 & 1,052 & 27 \\
\hline 13 & Rt & 1,312 & 1,078 & 234 & 722 & 765 & 43 & 248 & 278 & 30 & 166 & 163 & 3 \\
\hline 14 & Rt & 1,367 & 1,333 & 34 & 1,795 & 1,558 & 237 & 1,271 & 2,048 & 777 & 1,900 & 2,353 & 453 \\
\hline
\end{tabular}

*, difference in EMG amplitudes evoked by a patch stimulator and a conventional probe stimulator. ${ }^{\dagger}$, $\mathrm{P}$ value for the mean amplitudes between the two groups. Rt, right side; Lt, left side; V1, initial electromyography signal of the vagus nerve before surgical dissection; R1, initial electromyography signal of the recurrent laryngeal nerve upon initial identification; R2, electromyography signal of the recurrent laryngeal nerve after thyroidectomy; V2, electromyography signal of the vagus nerve after thyroidectomy; EMG, electromyography; SD, standard deviation.

stimulator, were developed using the same concept of stimulating dissecting instruments $(8,9)$. These attachable stimulators are more convenient in terms of preoperative instrument preparation and applicability to all metallic surgical instruments, and their nerve detection capabilities have been shown to be comparable to that of conventional stimulators $(6,8,9)$. However, there are some disadvantages to the attachable stimulators. A drawback of the magnetic attachable stimulator is its easy accidental detachment from the dissecting instrument, which can delay surgical procedures. The ring stimulator is bulky, and nerve stimulus cannot be delivered if the rubber ring becomes loosened from the surgical instrument. Lastly, these attachable stimulators are costly and not widely commercialized because they were developed specifically for IONM.

On the other hand, the patch stimulator used in our study is an adhesive nerve stimulator electrode commonly used for transcutaneous electrical nerve stimulation for therapeutic purposes, including pain relief and biofeedback therapies $(21,22)$. In our study, the patch stimulator showed 
Table 3 Comparison of different types of stimulators

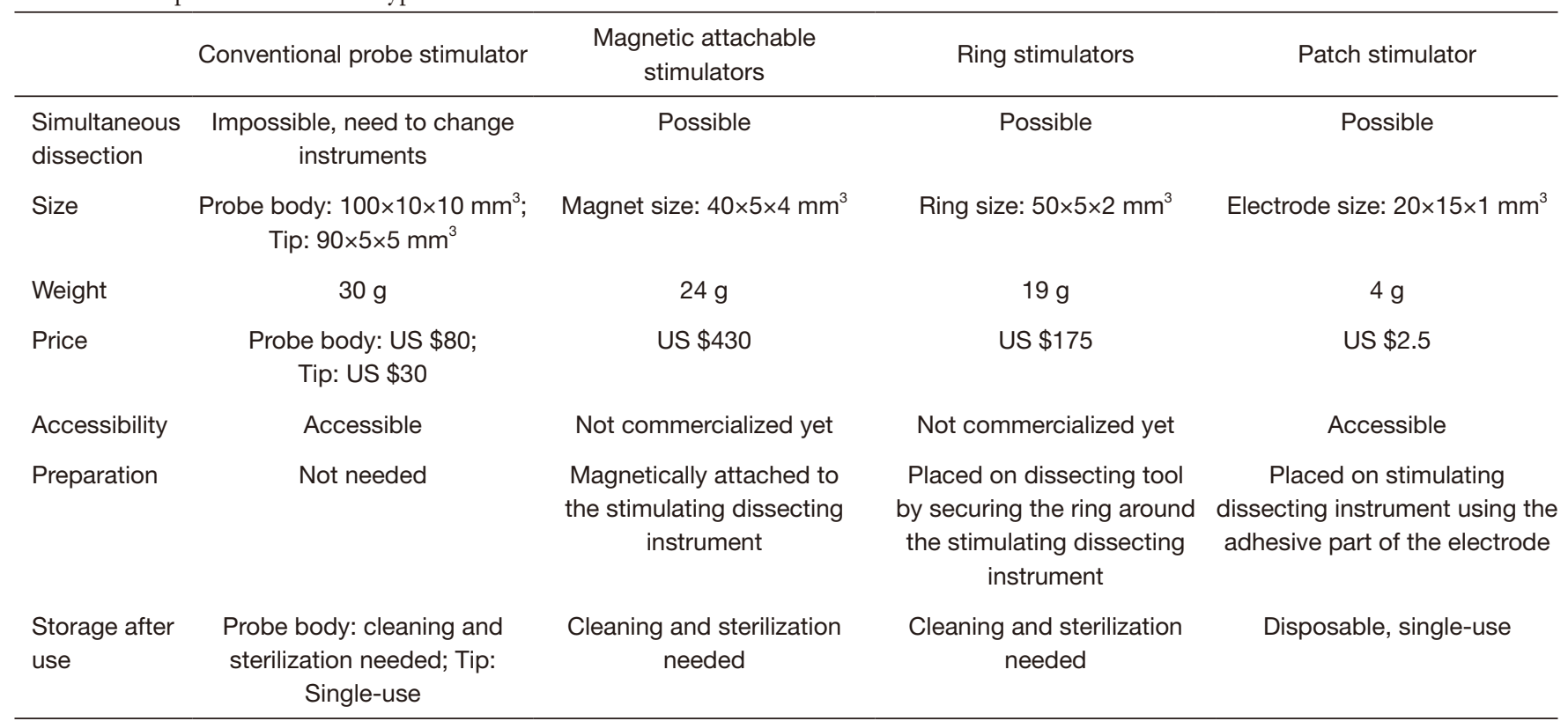

good conductivity compared to conventional stimulators, demonstrating its applicability for IONM during thyroid surgeries. Adhesive patch electrodes are small $(20 \times 15 \times$ $1 \mathrm{~mm}^{3}$ ), lightweight (4 grams) and can be easily applied to dissecting tools using the adhesive part of the patch stimulator. The adhesive makes accidental detachment from the surgical instruments less likely compared to other attachable stimulators, such as the magnetic attachable stimulator and the ring stimulator. Furthermore, the patch stimulators are disposable and inexpensive (US\$ 2.5) per single patch stimulator. There is no need to clean, disinfect, and reprocess them after each surgery, unlike reusable nerve stimulators, which require the same high level of care and maintenance as any other non-disposable surgical instrument (23). A comparison of the different types of stimulators is summarized in Table 3.

There are some limitations to using the patch stimulator approach. One limitation is that the patch stimulator can only be applied to one surgical instrument at a time. If a surgeon decides to apply the patch stimulator to another instrument, the task of taking the adhesive off the first instrument and applying it to another can be cumbersome and time-consuming. However, because most surgeons usually use one stimulating dissecting instrument per surgery, one patch stimulator usually is sufficient. Another limitation of using the patch stimulator is that, like other stimulating dissecting instruments, the surgical instruments are not insulated. If the tip of the instruments comes in contact with structures other than the nerves, the electrical current will most likely be inadequately dispersed during stimulation, and as a result, the appropriate voltage needed for identifying the nerves may be insufficient. To solve this potential problem, a higher voltage of stimulus (2-3 mA) could be given to identify the nerves, and extra care should be made to avoid contact with surrounding structures during nerve stimulation. To overcome this issue, new surgical instruments that are entirely insulated except for the tip could be developed for effective nerve stimulation. We took care not to allow the monopolar or energy-based device to make contact with the stimulating dissecting instrument. Doing so could result in a blown fuse in the nerve monitoring system when the electric current is transferred to the system. Lastly, this study has a small sample size. Nevertheless, we showed the feasibility of the use of patch stimulators for IONM, and anticipate that this study will be a basis for further studies with larger sample size.

\section{Conclusions}

EMG signals of the VN and RLN stimulated by the patch stimulator were comparable to those stimulated by the conventional stimulator. The patch stimulator allows the surgeon to simultaneously dissect and stimulate the nerves 
and can be safely and effectively used for IONM during thyroid surgery.

\section{Acknowledgments}

Authors would like to thank Jun Hwa Kang for the photographs. Funding: This research was supported by a grant of PatientCentered Clinical Research Coordinating Center funded by the Ministry of Health \& Welfare, Republic of Korea (grant number: HI19C0481, HC19C0103).

\section{Footnote}

Reporting Checklist: The authors have completed the STROBE reporting checklist. Available at https://dx.doi. org/10.21037/gs-21-327

Data Sharing Statement: Available at https://dx.doi. org/10.21037/gs-21-327

Peer Review File: Available at https://dx.doi.org/10.21037/ gs-21-327

Conflicts of Interest: All authors have completed the ICMJE uniform disclosure form (available at https://dx.doi. org/10.21037/gs-21-327). The authors have no conflicts of interest to declare.

Ethical Statement: The authors are accountable for all aspects of the work in ensuring that questions related to the accuracy or integrity of any part of the work are appropriately investigated and resolved. The study was conducted in accordance with the Declaration of Helsinki (as revised in 2013). The study was approved by Institutional Review Board of the Seoul Metropolitan Government Seoul National University Boramae Medical Center (IRB number: 30-2021-53) and individual consent for this retrospective analysis was waived.

Open Access Statement: This is an Open Access article distributed in accordance with the Creative Commons Attribution-NonCommercial-NoDerivs 4.0 International License (CC BY-NC-ND 4.0), which permits the noncommercial replication and distribution of the article with the strict proviso that no changes or edits are made and the original work is properly cited (including links to both the formal publication through the relevant DOI and the license). See: https://creativecommons.org/licenses/by-nc-nd/4.0/.

\section{References}

1. Dralle H, Sekulla C, Haerting J, et al. Risk factors of paralysis and functional outcome after recurrent laryngeal nerve monitoring in thyroid surgery. Surgery 2004;136:1310-22.

2. Baek SK, Lee K, Oh D, et al. Efficiency of intraoperative neuromonitoring on voice outcomes after thyroid surgery. Auris Nasus Larynx 2017;44:583-9.

3. Chiang FY, Lu IC, Chen HC, et al. Intraoperative neuromonitoring for early localization and identification of recurrent laryngeal nerve during thyroid surgery. Kaohsiung J Med Sci 2010;26:633-9.

4. Chiang FY, Lu IC, Kuo WR, et al. The mechanism of recurrent laryngeal nerve injury during thyroid surgery-the application of intraoperative neuromonitoring. Surgery 2008;143:743-9.

5. Dionigi G, Bartolo V, Rizzo AG, et al. Improving Safety of Neural Monitoring in Thyroid Surgery: Educational Considerations in Learning New Procedure. J Endocr Surg 2018;18:21-36.

6. Chiang FY, Lu IC, Chang PY, et al. Stimulating dissecting instruments during neuromonitoring of RLN in thyroid surgery. Laryngoscope 2015;125:2832-7.

7. Shin SC, Sung ES, Choi SW, et al. Feasibility and safety of nerve stimulator attachment to energy-based devices: A porcine model study. Int J Surg 2017;48:155-9.

8. Kim J, Moon HJ, Chai YJ, et al. Feasibility of Attachable Ring Stimulator for Intraoperative Neuromonitoring during Thyroid Surgery. Int J Endocrinol 2020;2020:5280939.

9. Sung ES, Lee JC, Shin SC, et al. Development of a Novel Detachable Magnetic Nerve Stimulator for Intraoperative Neuromonitoring. World J Surg 2018;42:137-42.

10. Randolph GW, Dralle H; International Intraoperative Monitoring Study Group, et al. Electrophysiologic recurrent laryngeal nerve monitoring during thyroid and parathyroid surgery: international standards guideline statement. Laryngoscope 2011;121 Suppl 1:S1-16.

11. Barczyński M, Konturek A, Cichoń S. Randomized clinical trial of visualization versus neuromonitoring of recurrent laryngeal nerves during thyroidectomy. Br J Surg 2009;96:240-6.

12. Dralle H, Lorenz K. Intraoperative neuromonitoring of thyroid gland operations : Surgical standards and aspects of expert assessment. Chirurg 2010;81:612-9.

13. Dionigi G, Alesina PF, Barczynski M, et al. Recurrent laryngeal nerve injury in video-assisted thyroidectomy: 
lessons learned from neuromonitoring. Surg Endosc 2012;26:2601-8.

14. Sun H, Zanghì GN, Freni F, et al. Continuous and intermitted nerve monitoring in thyroid surgery: two complementary devices. Gland Surg 2018;7:S80-1.

15. Kim HY, Chai YJ, Barczynski M, et al. Technical Instructions for Continuous Intraoperative Neural Monitoring in Thyroid Surgery. J Endocr Surg 2018;18:61-78.

16. Schneider R, Machens A, Sekulla C, et al. Superiority of continuous over intermittent intraoperative nerve monitoring in preventing vocal cord palsy. Br J Surg 2021;108:566-73.

17. Schneider R, Sekulla C, Machens A, et al. Postoperative vocal fold palsy in patients undergoing thyroid surgery with continuous or intermittent nerve monitoring. Br J Surg 2015;102:1380-7.

18. Marin Arteaga A, Peloni G, Leuchter I, et al. Modification of the Surgical Strategy for the Dissection of the Recurrent Laryngeal Nerve Using Continuous Intraoperative Nerve
Monitoring. World J Surg 2018;42:444-50.

19. Terris DJ, Chaung K, Duke WS. Continuous Vagal Nerve Monitoring is Dangerous and Should not Routinely be Done During Thyroid Surgery. World J Surg 2015;39:2471-6.

20. Lombardi CP, De Waure C, Mariani M, et al. Efficacy of continuous neuromonitoring in thyroid surgery: preliminary report of a single-center experience. Gland Surg 2019;8:336-42.

21. Dos Reis JN, Mello MF, Cabral BH, et al. EMG biofeedback or parasacral transcutaneous electrical nerve stimulation in children with lower urinary tract dysfunction: A prospective and randomized trial. Neurourol Urodyn 2019;38:1588-94.

22. Xu J, Sun Z, Wu J, et al. Peripheral Nerve Stimulation in Pain Management: A Systematic Review. Pain Physician 2021;24:E131-52.

23. Croke L. Guideline for care and cleaning of surgical instruments. AORN J 2020;112:P9-P11.
Cite this article as: Oh MY, Lee JM, Lee MH, Choi HS, Kim J, Hwang KT, Chai YJ. Application of patch stimulator for intraoperative neuromonitoring during thyroid surgery: maximizing surgeon's convenience. Gland Surg 2021;10(8):23782385. doi: $10.21037 /$ gs-21-327 